\title{
Improved Regional Analysis of Oxygen-Enhanced Lung MR Imaging Using Image Registration
}

\author{
Josephine H. Naish ${ }^{1}$, Geoffrey J.M. Parker ${ }^{1}$, Paul C.Beatty ${ }^{1}$, Alan Jackson ${ }^{1}$, \\ John C. Waterton ${ }^{2}$, Simon S. Young ${ }^{3}$, and Chris J. Taylor ${ }^{1}$ \\ 1 Imaging Science and Biomedical Engineering, University of Manchester, Manchester, UK \\ 2 Global Sciences and Information, AstraZeneca, Alderley Park, Macclesfield,UK \\ 3 AstraZeneca R\&D Charnwood, Bakewell Road, Loughborough, UK
}

\begin{abstract}
Oxygen enhanced MR imaging of the lung is a promising technique for monitoring a range of pulmonary diseases but regional analysis is hampered by lung motion and volume changes due to breathing. We have developed an image registration method to improve the quantitative regional analysis of both static and dynamic oxygen-enhanced pulmonary MRI. Images were acquired using a HASTE sequence at $1.5 \mathrm{~T}$ for five normal volunteers alternately breathing air and $100 \%$ oxygen. Static images were used to calculate regional changes in relaxation rate between breathing air and oxygen which is directly related to the increase in the dissolved oxygen concentration. Dynamic scans were used to calculate regional maps of oxygen wash-in and wash-out time constants. The method provided significant improvements in the both the static and the dynamic analysis. This may provide improved information in the regional assessment of chronic obstructive lung diseases.
\end{abstract}

\section{Introduction}

Oxygen-enhanced MR imaging of the lung has been demonstrated in healthy volunteers and in patients with pulmonary diseases [1|23.4]. Molecular oxygen is paramagnetic and so acts as a contrast agent when dissolved in parenchymal plasma due to its effect on $T_{1}$. Breathing $100 \%$ oxygen results in an increase in the concentration of dissolved oxygen in the lung tissue producing a corresponding decrease in $T_{1}$ which can be detected as a regional signal intensity increase in a $T_{1}$-weighted image. Unlike hyperpolarized gas MRI, which visualizes directly the air spaces in the lung, oxygen-enhanced MRI provides an indirect assessment of lung ventilation that may also depend on available tissue surface area and oxygen clearance by the blood. The method has the advantage over hyperpolarized gas MRI of requiring little specialized equipment so being more straightforward to implement in a clinical setting.

While a number of studies have demonstrated regional ventilation using oxygenenhanced MRI, a pixel-by-pixel analysis is made difficult by changes in size and shape of the lungs due to breathing. To date, little has been reported on characterizing regional time courses of oxygen wash-in and wash-out [5,6]. Breath-holding has been used in static ventilation studies [78] to overcome problems due to breathing motion but it can be uncomfortable for patients and it is difficult to perform in a reproducible manner. Various alternatives to breath-holding, designed to allow data acqisition during free 
breathing, have also been suggested. These include time-averaging sets of images [2 5], retrospective respiratory gating by selecting images for which the diaphragm position matches [1.9], respiratory triggering [710] and correlation analysis [811]. In this study we have developed an image registration method to correct for breathing motion which leads to significant improvements in the determination of both regional oxygen induced changes in $T_{1}$ and in the time course of regional signal intensity change during oxygen wash-in and wash-out.

\section{Methods}

\subsection{Imaging}

Imaging was performed at $1.5 \mathrm{~T}$ on five normal volunteers (non-smokers aged 30-39). A half Fourier single shot turbo spin-echo (HASTE) sequence was used with 68 phase encoding steps and inter-echo spacing of 4ms, 128128 matrix, coronal section with slice thickness $10 \mathrm{~mm}$. Volunteers breathed air or $100 \%$ oxygen through an MR compatible Bain breathing system and tightly fitting face mask. Gas was supplied at 101/min using a standard anaesthesia trolley.

Static $T_{1}$ measurements were performed using a saturation recovery HASTE sequence with nine saturation times $(T S)$ between $100 \mathrm{~ms}$ and $3.5 \mathrm{~s}$. Five images were collected for each saturation time to enable averaging over the cardiac cycle. Saturation recovery (SR) was chosen in preference to inversion recovery (IR) because of the shorter total imaging time (approximately $2.5 \mathrm{~min}$ for the set of 45 images). Dynamic image acquisitions were performed using an IR HASTE sequence during oxygen wash-in and wash-out. An inversion time of $720 \mathrm{~ms}$ was chosen to approximately null the signal while breathing air [3]. Dynamic images were acquired every 3.5s for a total of $200 \mathrm{~s}$ and the gas supply was switched from medical air to $100 \%$ oxygen (or vice-versa) after the 10th image in the set.

\subsection{Image Registration}

During breathing the lung is deformed mainly in the longitudinal direction due to motion of the diaphragm and, to a lesser extent, expansion of the chest cage. The registration procedure that we have adopted is based on this observation and consists of two steps. The first step utilises an active shape model (ASM) [12] to allow an automated identification of the lung outline. An ASM combines a point distribution model (PDM) with an image search and enables the automatic detection of structures in a wide variety of both medical and non-medical images. In the second step the lung outline is registered to a reference using a 1D linear transformation.

The first ten images from a set of 116 dynamic scans ( 58 for oxygen wash-in and 58 for oxygen wash-out)were manually marked up to build the ASM. Ten points were manually marked along the diaphragm and a further twelve points were marked at landmarks around the lung outline; where possible these marked the intervertebral disks and the positions of the ribs. The ten points along the diaphragm were then re-sampled at equidistant points along the curve using an Akima spline interpolation [13]. The resulting 
set of 22 points for each of the ten images form the set of correspondences which are used to calculate the PDM. Over $99 \%$ of the variation in lung shape was described by the first two modes of variation of the PDM as illustrated in Fig. 10 The ASM search was used to locate the lung outline in the remaining 106 images in the dynamic set. An example search result is presented in Fig. 1
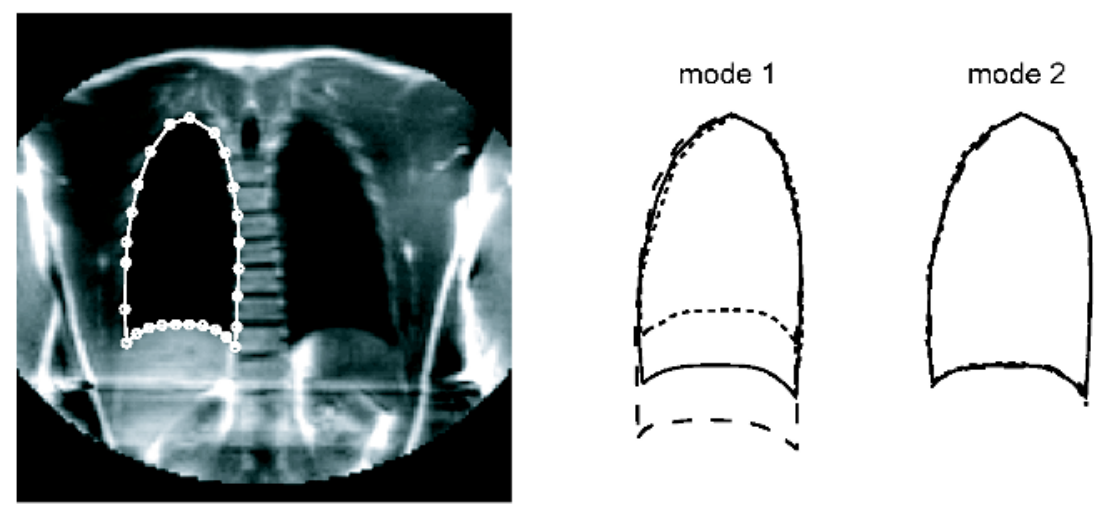

Fig. 1. An example image from a dynamic set showing the results of the active shape model search and the first two modes of variation of the active shape model. The solid line is the mean shape and the dashed and dotted lines are +2 s.d and -2 s.d respectively

The images were transformed onto a reference by re-sampling the lung outline at discrete horizontal pixel locations around the top edge of the lung and along the diaphragm and applying a simple linear transformation on each column of pixels. Signal intensity was sampled at off-pixel locations using linear interpolation and re-scaled according to the magnitude of the stretch to account for proton density variation during the breathing cycle.

The $T_{1}$ measurement SR HASTE images were registered by manually marking up all 45 images in each measurement set and transforming as described above. In this case it was not possible to build an ASM to minimize the user input because of the large variation in contrast between images with different $T S$.

\subsection{Analysis}

$T_{1}$ maps were produced from each set of 45 registered SR HASTE images by fitting on a pixel-by-pixel basis using an exponential of the form $S(T S)=A-B \exp \left(-T S / T_{1}\right)$ where $S(T S)$ is the pixel intensity at each $T S$ and $A$ and $B$ are constants. Oxygen washin and wash-out times were calculated from the dynamic IR HASTE sets of images by fitting an exponential to the dynamic signal intensity both in regions of interest and on a pixel-by-pixel basis in a smoothed set of images. 


\section{Results}

The ASM search successfully located the lung outline in all 116 dynamic images for three of the volunteers and in all but 3 of the 116 images for each of the other two volunteers. Virtually all of the shape variation is captured in the first mode, and this is mainly a simple linear stretch in the vertical direction as illustrated in Fig. 1.
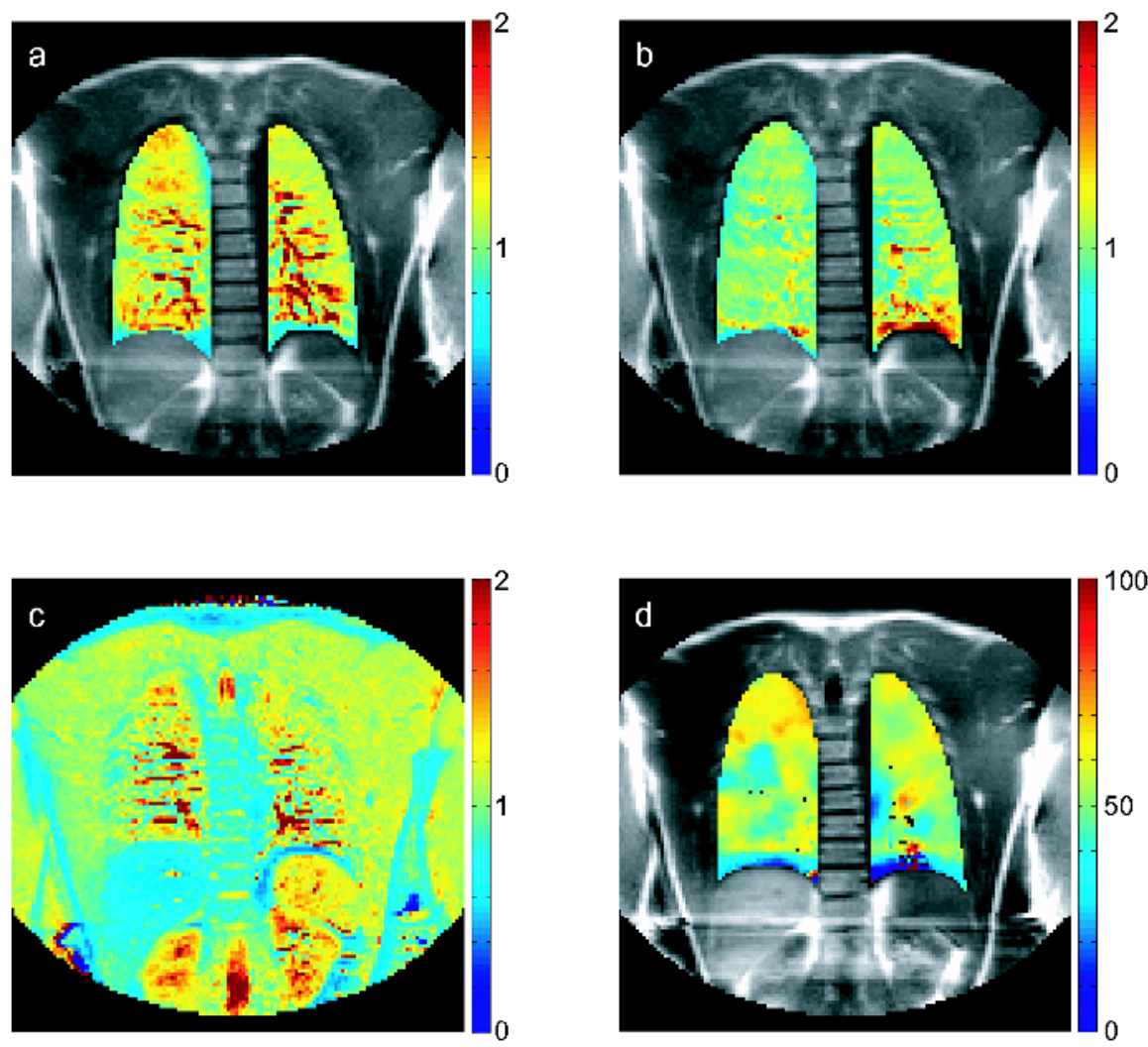

Fig. 2. a)-c) Example $T_{1}$ maps for a subject breathing medical air (a \& c) and 100\% oxygen (b). The maps in a \& b are calculated using the full set of registered images and the map in $c$ is calculated using a subset of images in which the right diaphragm position matches to \pm 1 pixel (retrospective gating). d) Example regional uptake time map. The scales for all four maps are in units of $s$.

In Fig. 2a \& b we present an example of matched $T_{1}$ maps for a volunteer breathing medical air and $100 \%$ oxygen. A reduction in $T_{1}$ on breathing oxygen is demonstrated across the whole of the lung. $T_{1}$ values averaged within the lung breathing air and oxygen are $1.27 \mathrm{~s}$ and $1.08 \mathrm{~s}$ respectively and are consistent with previously published values [2:14]. Figure $2 \mathrm{k}$ is a $T_{1}$ map calculated by selecting those images for which the 

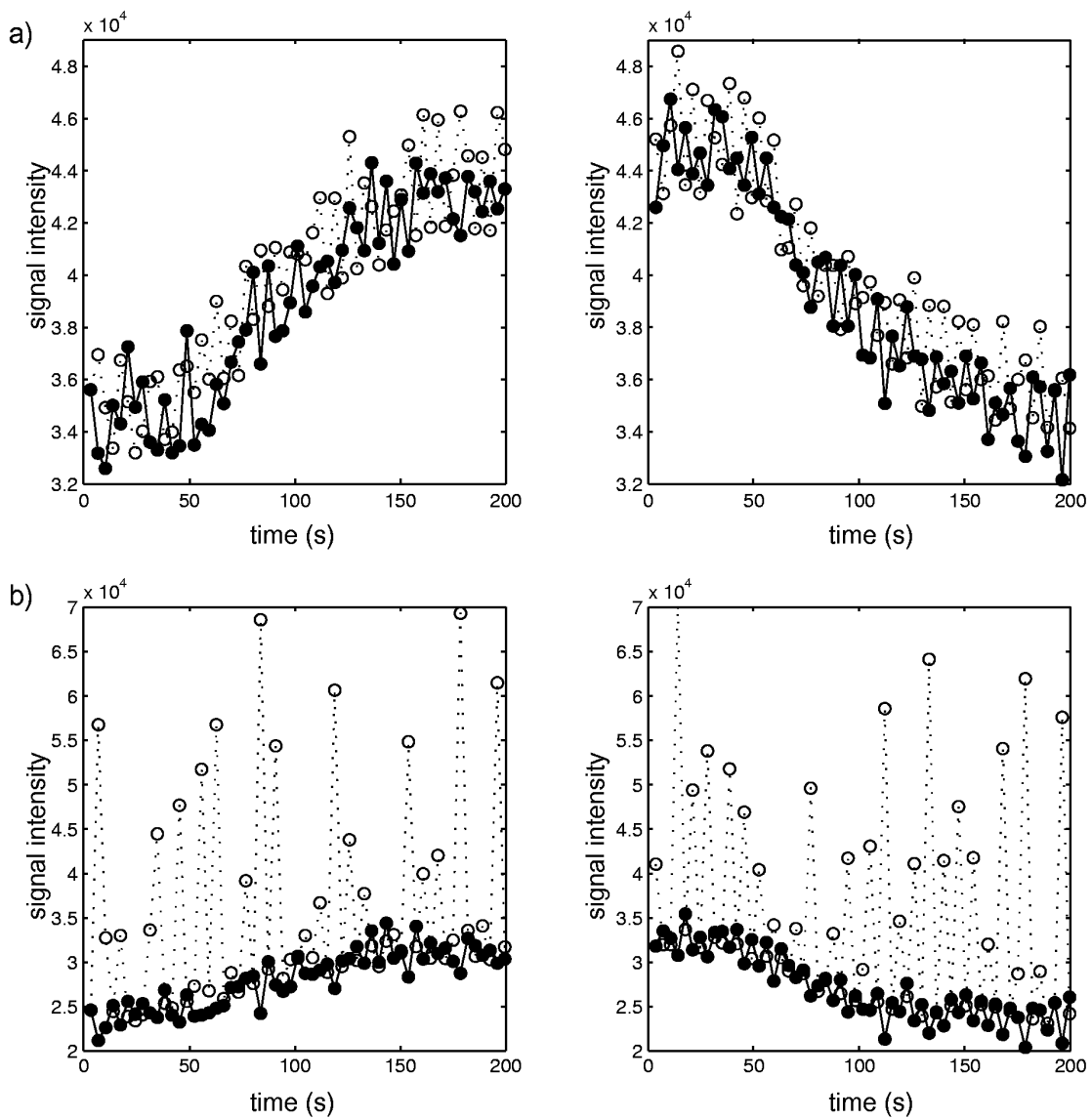

Fig. 3. Example dynamic wash-in and wash-out curves for a region of interest in a) the upper half of the right lung and b) the lower half of the right lung. The dotted lines (open symbols) are for raw images and the solid lines (filled symbols) are for registered images

right diaphragm matches to within 2 pixels (i.e. \pm 1 pixel from the mean position in the subset of images), which effectively forms a retrospectively gated map. The $T_{1}$ map calculated from the registered set of images shows comparatively clearer structure and a reduction in breathing artefact. The contribution of dissolved oxygen to the relaxation rate depends linearly on its concentration [14]. The difference in relaxation rate between breathing air and $100 \%$ oxygen, $1 / T_{1 \text { (oxygen) }}-1 / T_{1 \text { (air) }}$, is directly proportional to the additional dissolved oxygen on breathing $100 \%$ oxygen thus this technique allows a spatial mapping of the relative regional distribution of additional dissolved oxygen.

Signal intensity time courses are plotted in Fig. 3 for regions of interest defined in the upper and lower halves of the right lung. Registration leads to a small improvement in the upper ROI seen as a reduction in noise of the solid lines compared to the dotted lines. A more dramatic improvement can be seen for the lower ROI which is much more 
affected by the movement of the diaphragm during breathing. The results of fitting an exponential to the dynamic signal intensity in order to obtain oxygen wash-in and washout time constants for a ROI encompassing the whole of the left or the right lung are presented in Table 1 for the five volunteers. The mean wash-in times for the right and left lungs were $57 \pm 5$ s and $46 \pm 14$ s. The mean wash-out times for the right and left lungs were $38 \pm 14 \mathrm{~s}$ and $38 \pm 13 \mathrm{~s}$. An example regional wash-in time map, found by fitting the smoothed images on a pixel-by-pixel basis, is presented in Fig. $2 \mathrm{~d}$.

Table 1. Average wash-in and wash-out times in seconds for the right and left lungs of five normal volunteers

\begin{tabular}{ccccc}
\hline & \multicolumn{2}{c}{ wash-in } & \multicolumn{2}{c}{ wash-out } \\
subject & right & left & right & left \\
\hline 1 & 53.7 & 46.9 & 31.8 & 34.0 \\
2 & 58.7 & 21.9 & 27.0 & 37.7 \\
3 & 65.1 & 50.4 & 40.9 & 31.0 \\
4 & 54.5 & 50.6 & 61.5 & 61.2 \\
5 & 53.2 & 62.1 & 29.7 & 27.0 \\
\hline mean(s.d.) & $57(5)$ & $46(14)$ & $38(14)$ & $38(13)$ \\
\hline
\end{tabular}

\section{Discussion}

We have demonstrated an image registration method which results in significant improvements in the regional analysis of oxygen enhanced lung MR imaging. The increase in concentration of oxygen dissolved in the lung tissue as a result of breathing $100 \%$ oxygen leads to a shortening of the parenchymal $T_{1}$. A regional determination of change in relaxation rate directly corresponds to the regional increase in concentration of dissolved oxygen. The image registration method used therefore allows a pixel-by-pixel determination of relaxation rate change and hence the relative regional increase in dissolved oxygen.

Regional dynamic oxygen wash-in and wash-out analysis is also improved by the use of registration, particularly in the lower region of the lung which is most affected by diaphragm motion. There is some evidence that the wash-in is heterogeneous across the lung even in normal volunteers as indicated in Fig. 2 1 . Longer wash-in time constants seem to be present in the upper lobes which is consistent with what might be expected for a supine subject. The average time constants as presented in Table 1 are longer than those reported by Hatabu et al [5], possibly as a result of differences in the breathing apparatus used. In common with Hatabu et al, we observe shorter wash-out times compared to wash-in, probably due to dissolved oxygen being carried from the lungs in the blood and removed by tissues in other parts of the body. 
During breathing, the majority of the movement and volume change observed in the lung is as a result of the diaphragm motion with an additional smaller effect due to the expansion of the chest wall. This observation is also confirmed by the modes of variation of the ASM and led to the selection of a 1D linear model to describe the deformation. A limitation of this model is that it does not take account of movement out of the coronal plane, although this is expected to be small compared to the in-plane motion, and of limited significance due to the relatively large $(10 \mathrm{~mm})$ slice thickness used. In the present study we have investigated slices positioned posteriorly, further limiting out of plane motion. Another limitation of our study is that in rescaling the pixel signal intensity according to the magnitude of the stretch we are assuming that the total proton density within the lung remains constant over a breathing cycle. This may not be a valid assumption and in fact we observe a tendency to over-correct which may be due to an increase in blood volume in the lung at maximum inhalation. Methods which rely on some form of respiratory gating are less affected by out-of-plane movement and changes in proton density but suffer from remaining motion artefacts due to some remaining differences in diaphragm position. In the case of retrospective gating there is also a considerable loss of temporal resolution. An improvement to our method may be to combine it with respiratory triggering which would result in a relatively small loss of temporal resolution.

\section{Conclusions}

We have demonstrated that by using image registration we can improve the regional determination of both the change in relaxation rate on breathing $100 \%$ oxygen and the rate of change of signal intensity on switching from air to $100 \%$ oxygen. This results in an improved understanding of the regional change in oxygen concentration and the oxygen wash-in and wash-out rates which may be important in the regional evaluation of chronic obstructive pulmonary diseases.

\section{References}

1. Edelman RR, Hatabu H, Tadamura E, Li W, and Prasad PV. Noninvasive assessment of regional ventilation in the human lung using oxygen-enhanced magnetic resonance imaging. Nat Med, 2(11):1236-9, 1996.

2. Chen Q, Jakob PM, Griswold MA, Levin DL, Hatabu H, and Edelman RR. Oxygen enhanced mr ventilation imaging of the lung. Magma, 7(3):153-61, 1998.

3. Ohno Y, Hatabu H, Takenaka D, Adachi S, Van Cauteren M, and Sugimura K. Oxygenenhanced MR ventilation imaging of the lung: preliminary clinical experience in 25 subjects. AJR Am J Roentgenol, 177(1):185-94, 2001.

4. Ohno Y, Hatabu H, Takenaka D, Van Cauteren M, Fujii M, and Sugimura K. Dynamic oxygenenhanced MRI reflects diffusing capacity of the lung. Magn Reson Med, 47(6):1139-44, 2002.

5. Hatabu H, Tadamura E, Chen Q, Stock KW, Li W, Prasad PV, and Edelman RR. Pulmonary ventilation: dynamic MRI with inhalation of molecular oxygen. Eur J Radiol, 37(3):172-8, 2001.

6. Muller CJ, Schwaiblmair M, Scheidler J, Deimling M, Weber J, Loffler RB, and Reiser MF. Pulmonary diffusing capacity: assessment with oxygen-enhanced lung MR imaging preliminary findings. Radiology, 222(2):499-506, 2002. 
7. Stock KW, Chen Q, Morrin M, Hatabu H, and Edelman RR. Oxygen-enhanced magnetic resonance ventilation imaging of the human lung at 0.2 and 1.5 t. J Magn Reson Imaging, 9(6):838-41, 1999.

8. Loffler R, Muller CJ, Peller M, Penzkofer H, Deimling M, Schwaiblmair M, Scheidler J, and Reiser M. Optimization and evaluation of the signal intensity change in multisection oxygen-enhanced MR lung imaging. Magn Reson Med, 43(6):860-6, 2000.

9. Mai VM, Liu B, Li W, Polzin J, Kurucay S, Chen Q, and Edelman RR. Influence of oxygen flow rate on signal and $\mathrm{T}(1)$ changes in oxygen-enhanced ventilation imaging. J Magn Reson Imaging, 16(1):37-41, 2002.

10. Vaninbroukx J, Bosmans H, Sunaert S, Demedts M, Delcroix M, Marchal G, and Verschakelen J. The use of ECG and respiratory triggering to improve the sensitivity of oxygen-enhanced proton MRI of lung ventilation. Eur Radiol, 13(6):1260-5, 2003.

11. Mai VM, Tutton S, Prasad PV, Chen Q, Li W, Chen C, Liu B, Polzin J, Kurucay S, and Edelman RR. Computing oxygen-enhanced ventilation maps using correlation analysis. Magn Reson Med, 49(3):591-4, 2003.

12. Cootes TF, Taylor CJ, Cooper DH, and Graham J. Active Shape Models- their training and application. Comput Vis Image Underst, 61(1):38-59, 1995.

13. Akima $\mathrm{H}$. A new method of interpolation and smooth curve fitting based on local procedures. $J$ Acm, 17(4):589, 1970.

14. Ohno Y, Chen Q, and Hatabu H. Oxygen-enhanced magnetic resonance ventilation imaging of lung. Eur J Radiol, 37(3):164-71, 2001. 